\title{
Imbalance of tumor necrosis factor- $\alpha$, interleukin-8 and interleukin-10 production evokes barrier dysfunction, severe abdominal symptoms and psychological disorders in patients with irritable bowel syndrome-associated diarrhea
}

\author{
YANBO ZHEN, CHUANLIAN CHU, SHIQING ZHOU, MING QI and RONGHUA SHU \\ Department of Gastroenterology, Jinan Central Hospital, Jinan, Shandong 250013, P.R. China
}

Received April 30, 2014; Accepted January 30, 2015

DOI: $10.3892 / \mathrm{mmr} .2015 .4079$

\begin{abstract}
The present study aimed to explore the correlation between cytokine expression of tumor necrosis factor $\alpha$ (TNF- $\alpha$ ), interleukin (IL)-8 and IL-10 with occludin production, abdominal symptoms and psychological factors in patients with irritable bowel syndrome-associated diarrhea (IBS-D). A total of 42 IBS-D patients and 20 healthy controls were included, which were recruited from QiLu Hospital in China. ELISA and immunohistochemical analysis were performed for evaluating the cytokines (TNF- $\alpha$, IL- 8 and IL-10) and occludin protein levels in the peripheral blood mononuclear cells (PBMCs) of all subjects. In addition, the abdominal symptoms and psychological status were assessed in IBS-D patients. Levels of TNF- $\alpha$ and IL- 8 in the PBMCs of patients with IBS-D were significantly higher than those in the controls $(\mathrm{P}<0.001$ and $\mathrm{P}=0.007$, respectively), while IL-10 levels were significantly reduced in patients with IBS-D $(\mathrm{P}=0.047)$. Occludin production was significantly reduced in patients with IBS-D as compared with that in the controls $(\mathrm{P}<0.001)$. In patients with IBS-D, levels of TNF- $\alpha$ and IL- 8 were negatively correlated with occludin levels $(\mathrm{r}=-0.34, \mathrm{P}=0.028 ; \mathrm{r}=-0.52$, $\mathrm{P}<0.001$, respectively). IL-10 showed a negative correlation with occludin production $(\mathrm{r}=0.05, \mathrm{P}=0.748)$. Furthermore, TNF- $\alpha$, IL- 8 and IL-10 levels were significantly correlated with symptoms scores $(\mathrm{r}=0.74, \mathrm{P}<0.001 ; \mathrm{r}=0.55, \mathrm{P}<0.001 ; \mathrm{r}=-0.80$, $\mathrm{P}<0.001$, respectively) in patients with IBS-D. Within the IBS-D group, TNF- $\alpha$ expression was significantly increased in patients with a self-rating depression scale (SDS) score $\geq 50$ $(\mathrm{P}=0.004)$ as compared with that in patients with an SDS score $<50$. Furthermore, IL-8 was significantly increased in IBS-D patients with a self-rating anxiety scale (SAS) or SDS score
\end{abstract}

Correspondence to: Dr Yanbo Zhen, Department of Gastroenterology, Jinan Central Hospital, 105 Jiefang Road, Jinan, Shandong 250013, P.R. China

E-mail: yanbo0213y@gmail.com

Key words: irritable bowel syndrome, cytokines, occludin, abdominal symptoms, psychological factor $\geq 50(\mathrm{P}=0.016, \mathrm{P}=0.008$, respectively) as compared with that in patients scoring $<50$. In conclusion, the results of the present study suggested that in IBS-D, an imbalance of cytokine production evoked colonic epithelial barrier dysfunction, abdominal symptoms and psychological disorders.

\section{Introduction}

Irritable bowel syndrome (IBS) is a functional gastrointestinal disorder with symptoms including abdominal pain, frequent diarrhea, constipation and altered bowel habits (1). Based on the predominant symptoms, IBS is classified as IBS-associated diarrhea (IBS-D), constipation-predominant IBS (IBS-C) and IBS with alternating stool pattern (IBS-A) (2). IBS is a common clinical problem and has affected 8 to $20 \%$ of the general population worldwide (3). To date, great progress has been made in exploring the mechanism underlying IBS.

One mechanism underlying the pathophysiology of IBS is based on changes in immune activity. The imbalance of pro- and anti-inflammatory cytokines contributes to the development of IBS (4). An accumulation of the pro-inflammatory cytokine tumor necrosis factor $\alpha$ (TNF- $\alpha$ ) and a decreased production of interleukin 10 (IL-10) have been reported in IBS patients $(4,5)$. Interleukin $8(\mathrm{IL}-8)$ is a modulator in recruitment and activation of polymorphonuclear neutrophils. The protein levels of IL- 8 were found to be elevated during the development of IBS (6). In addition, proteasome-mediated protein degradation may be another factor leading to IBS. Furthermore, increasing occludin degradation was shown to contribute to the development of IBS (7). However, few studies have focused on the association between systemic cytokine expression and tight junction proteins in IBS.

The role of psychological factors in IBS is being increasingly recognized. IBS patients commonly show psychological symptoms, including anxiety, depression and fatigue $(8,9)$. In one study, patients with IBS with increased fatigue and depression were shown to have an elevated mass of mast cells in the caecal mucosa (10). A significant association was made between the severity of psychological symptoms and the cellularity of the lamina propria in patients with IBS. To date, the association between cytokine expression and psychological factors has not been clearly determined. 
The present study investigated the expression of the cytokines TNF- $\alpha$, IL-8 and IL-10, as well as occludin in IBS-D patients and healthy controls. In addition, patients with IBS-D completed a self report questionnaire and psychological assessment, which were used for the correlation of cytokine profiles with occludin protein production as well as abdominal and psychological symptoms in patients with IBS-D.

\section{Materials and methods}

Study population. The present study was approved by the Clinical Research Ethics Committee of QiLu Hospital of Shandong University in China and all participants or their parents signed informed consent forms prior to enrolment in the study.

A total of 42 patients with IBS-D were recruited from gastroenterology clinics at QiLu Hospital, including 15 males (mean age, 36.4 \pm 6.9 years; range, 20-57 years and 27 females (mean age, 38.1 \pm 5.9 years; range, 18-56 years) (Table I). All patients had IBS symptoms corresponding to the Rome III criteria, including abdominal discomfort or pain (11). Symptoms were required to be present for the previous six months and persist for at least three months. IBS patients undergoing more than three bowel movements per day and loose stool forms were diagnosed with IBS-D based on the Bristol Stool Form Scale (12). Patients with organic gastrointestinal disorders and acute gastrointestinal infection and organic disease (including diabetes mellitus, hepatic or renal dysfunction, and thyroid disease) were excluded. The patient cohort was selected following positive diagnosis using colonoscopy, biopsy, abdominal ultrasonography and other clinical or laboratorial tests, including blood and stool analyses followed by assessment of erythrocyte sedimentation rate, C-reactive protein, liver and renal function, thyroid function, fasting plasma glucose, calcium and electrolytes.

In addition, 20 healthy asymptomatic controls were recruited from the Department of Health Examination of QiLu Hospital, including eight males (mean age, $37.9 \pm 7.8$ years; range, 21-59 years) and 12 females (mean age, 35.1 \pm 8.1 years; range, 19-60 years) (Table I). The controls were not on any medication for gastrointestinal disease and had a normal body mass index.

Isolation and culture of peripheral blood mononuclear cells. A amount of $5 \mathrm{ml}$ peripheral blood were extracted from the healthy individuals and IBS-D patients following fasting for $12 \mathrm{~h}$. Isolation of peripheral blood mononuclear cells (PBMCs) was performed by density gradient centrifugation at 2,000 $\mathrm{xg}$ for $20 \mathrm{~min}$. Blood was collected into sterile heparinized tubes and mixed with $5 \mathrm{ml}$ RPMI 1640 (Gibco-BRL, Invitrogen Life Technologies, Carlsbad, CA, USA). The diluted blood was treated with $5 \mathrm{ml}$ Ficoll-Paque (Sigma-Aldrich, St Louis, MO, USA) and centrifuged at 2,000 $\mathrm{xg}$ for $20 \mathrm{~min}$. The PBMCs were then washed twice with phosphate-buffered saline (PBS) and RPMI 1640 media (Gibco-BRL). The viability of PBMCs was detected by trypan blue (Gibco-BRL) staining.

Cell culture and ELISA. PBMCs were resuspended to achieve a density of $1 \times 10^{6}$ cells $/ \mathrm{ml}$ in RPMI 1640 medium with $10 \%$ fetal bovine serum, $100 \mathrm{U} / \mathrm{ml}$ penicillin (Beyotime Institute of Biotechnology, Haimen, China) and $100 \mu \mathrm{g} / \mathrm{ml}$ streptomycin (Beyotime Institute of Biotechnology). PBMCs were cultured at $37^{\circ} \mathrm{C}$ in a humidified $5 \% \mathrm{CO}_{2}$ atmosphere for $24 \mathrm{~h}$. Cell-free supernatants were obtained following centrifugation at $3,000 \mathrm{xg}$ for $10 \mathrm{~min}\left(4^{\circ} \mathrm{C}\right)$ and stored at $-80^{\circ} \mathrm{C}$ for further investigation. The expression of TNF- $\alpha$, IL- 8 and IL-10 in PBMCs was measured using ELISA kits (Peprotech, Rocky Hill, NJ, USA) according to the manufacturer's instructions. Optical density (OD) was measured at a wavelength of $450 \mathrm{~nm}$ and the reference wavelength was corrected to $590 \mathrm{~nm}$, using an automated ELISA reader (ELx800; BioTek, Winooski, VA, USA). The limit of sensitivity of the assays was $2 \mathrm{pg} / \mathrm{ml}$.

Immunohistochemical analysis of occludin. Tissue samples from the rectosigmoid colon were obtained from all participants. Sections of colonic biopsies were fixed in $4 \%$ paraformaldehyde (Damao Chemical Reagent Factory, Tianjin, China) for $2 \mathrm{~h}$ at room temperature, embedded in optimum cutting temperature (OCT) compound (Fisher Thermo Scientific, Waltham, MA, USA) and subsequently frozen at $-80^{\circ} \mathrm{C}$. The sections of frozen colonic tissues $(4 \mu \mathrm{m})$ were mounted on glass slides and air-dried for $30 \mathrm{~min}$, then fixed with acetone (Big Alum Chemical Reagent Factory) $\left(10 \mathrm{~min}, 4^{\circ} \mathrm{C}\right)$ and washed in PBS. Sections were treated with $3 \% \mathrm{H}_{2} \mathrm{O}_{2}$ in methanol (Big Alum Chemical Reagent Factory) for $15 \mathrm{~min}$ and non-specific binding was blocked with 5\% goat serum (Intergen, Purchase, NY, USA) for $4 \mathrm{~h}$ at room temperature. Then sections were incubated with rabbit monoclonal anti-occludin antibody (1:100 dilution; Zymed, Invitrogen Life Technologies) overnight at $4^{\circ} \mathrm{C}$ and horseradish peroxidase (HRP)-conjugated goat anti-rabbit immunoglobulin (Ig)G antibody (1:5,000; ZSJQ-BIO, Beijing, China) for $2 \mathrm{~h}$ at room temperature. The slides were washed in PBS and stained with diaminobenzidene (DAB; Amresco LLC, Solon, OH, USA). The microphotographs were examined under a microscope (BX-51; Olympus Corp., Tokyo, Japan).

Western blot analysis. For western blot analysis, biopsy specimens from the rectosigmoid colon were immediately stored at $-80^{\circ} \mathrm{C}$. Protein was extracted from $100-\mathrm{mg}$ colonic samples by homogenization in ice-cold lysate buffer (20 mmol/l Tris-HCl, pH 7.5; $100 \mathrm{mmol} / \mathrm{l} \mathrm{NaCl} ; 0.5 \mathrm{mmol} / \mathrm{l}$ EDTA; $1 \mathrm{mmol} / 1 \mathrm{PMSF} ; 1 \mathrm{mmol} / \mathrm{l}$ DTT; $2 \mathrm{mmol} / 1$ sodium orthovanadate; $0.5 \%$ Nonider $\mathrm{P} 40$; and $0.1 \%$ protease inhibitor cocktail; all from Sigma-Aldrich) for $30 \mathrm{~min}$ at $4^{\circ} \mathrm{C}$ and centrifugation at $15,000 \mathrm{x}$ g for $15 \mathrm{~min}$ at $4^{\circ} \mathrm{C}$. The protein content was determined by Pierce bicinchoninic acid (BCA) protein assay kit (Fisher Thermo Scientific). Protein samples $(25 \mathrm{mg})$ were separated using $10 \%$ SDS-PAGE and transferred to nitrocellulose membranes (Pall Corporation, Dreieich, Germany). Membranes were blocked in 5\% nonfat milk (BD Biosciences, San Jose, CA, USA) for $1 \mathrm{~h}$ at room temperature. The membranes were incubated with rabbit monoclonal anti-occludin (1:50 dilution; Zymed) and monoclonal anti- $\beta$-actin (1:1,000; Kangtai Biological Products Co., Ltd., Shanghai, China) overnight at $4^{\circ} \mathrm{C}$. The membranes were then incubated with HRP-conjugated goat anti-rabbit IgG antibody (1:5,000; Biokangtai) for $1 \mathrm{~h}$ at room temperature. The expression intensity was determined by calculating the ratio of integrated optical density (IOD) between occludin and $\beta$-actin (Santa Cruz Biotechnology, Dallas, TX, USA). 
Table I. Basic characteristics of patients and healthy controls.

\begin{tabular}{lccccr}
\hline & \multicolumn{3}{c}{ Patients } & \multicolumn{2}{c}{ Controls } \\
\cline { 2 - 3 } Gender & Male & Female & Male & Female \\
\hline Number & 15 & 27 & 8 & 12 \\
Age $^{\mathrm{a}}$ (years) & $36.4 \pm 6.9$ & $38.1 \pm 5.9$ & $37.9 \pm 7.8$ & $35.1 \pm 8.1$ \\
Range (years) & $20-57$ & $18-56$ & $21-59$ & $19-60$ \\
\hline
\end{tabular}

${ }^{\mathrm{a}}$ Expressed as the mean \pm standard deviation.

Symptom questionnaire. In order to evaluate the severity of the symptoms, a self-validated report questionnaire based on the Rome III criteria was developed for the IBS-D patients according to previous studies $(13,14)$. The questionnaire items mainly focused on the intensity and frequency of abdominal symptoms, including abdominal pain, incomplete emptying following bowel movement, three or more bowel movements per day, loose, mushy or watery stools, and urgency to move bowels. The intensity of abdominal symptoms was graded according to the criteria as follows: 0 , Absence of symptoms; 1 , mild symptoms; 2, moderate symptoms, which do not affect day-to-day activities; and 3, severe symptoms, which impaired day-to-day activities. The frequency scoring criterion was 0 , no onset; 1 , one to three times per week; 2, four to six times per week; and 3, every day. The total scores ranging from 0 to 15 were calculated as the sum of each item score of abdominal symptoms.

Psychological assessment. The Zung self-rating anxiety scale (SAS) and the self-rating depression scale (SDS) were applied to IBS-D patients according to the previous studies with slight modifications $(15,16)$. For SDS or SAS rating, questionnaires including 20 items were filled in by the patients to rate their perceived moods and feelings. The scoring for each item was graded according to the four quantitative terms: 1, a percentage of the time; 2 , some of the time; 3 , a good part of the time; 4, most of the time. The scoring for SAS or SDS was performed using a multiple choice questionnaire with positive and negative answering options. Initial scores were calculated as follows: Scores obtained from the 20 items of the SDS or SAS questionnaires were summarized and multiplied by 1.25 . The final score was rounded to the nearest whole score. A score $<50$ was considered to be normal, a score of 50-74 was defined to indicate a mild disorder, a score of 75-87 indicated a moderate disorder and a score $>88$ indicated a severe disorder. In the present study a score $\geq 50$ on the anxiety or depression scale was regarded to indicate psychological distress.

Statistical analysis. Data were analyzed by SPSS 16.0 (SPSS Inc., Chicago, IL, USA) and values were expressed as the mean \pm standard deviation. The independent-sample t-test was applied for assessing the difference in cytokine expression, IOD of occludin, abdominal symptoms scores and psychological scores between IBS-D patients and normal controls. The correlation among the variables was evaluated by Pearson correlation. $\mathrm{P}<0.05$ was considered to indicate statistically significant differences between values.

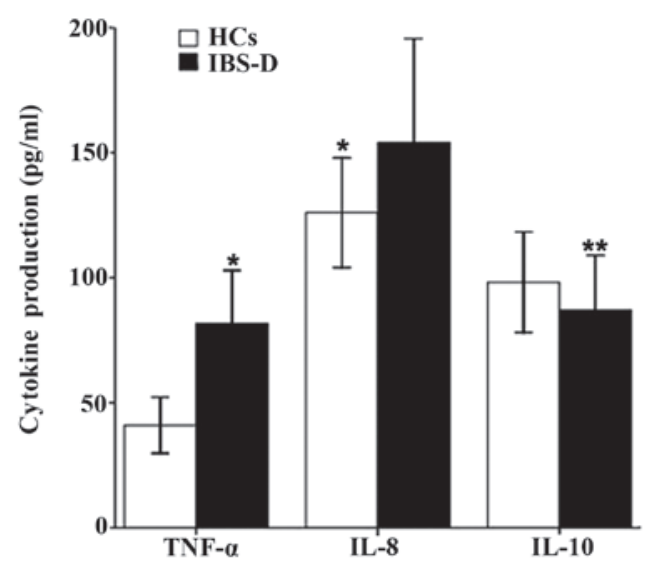

Figure 1. Comparison of cytokine production in peripheral blood mononuclear cells from healthy controls (HCs) and patients with IBS-D by ELISA. Values are expressed as the mean \pm standard deviation. ${ }^{*} \mathrm{P}<0.01,{ }^{* * *} \mathrm{P}<0.05$, as compared with the healthy controls. TNF- $\alpha$, tumor necrosis factor alpha; IL, interleukin; IBS-D, irritable bowel syndrome with diarrhea; HC, healthy control.

\section{Results}

Comparison of cytokine levels in the IBS-D and control groups. Overall, the TNF- $\alpha$ and IL- 8 levels $(81.51 \pm 22.18$ vs. $41.77 \pm 10.74 \mathrm{pg} / \mathrm{ml}, \mathrm{P}<0.001 ; 152.21 \pm 38.10$ vs. $126.19 \pm 24.12$ $\mathrm{pg} / \mathrm{ml}, \mathrm{P}=0.007)$ were significantly higher in IBS-D patients than those in healthy controls (Fig. 1). However, IBS-D patients showed decreased IL-10 production compared with that in controls $(87.11 \pm 22.46 \mathrm{vs} .98 .54 \pm 16.43 \mathrm{pg} / \mathrm{ml}, \mathrm{P}=0.047)$ (Fig. 1).

Comparison of occludin expression between IBS-D and control groups. Immunohistochemical analysis of occludin is shown in Fig. 2. Occludin was primarily present on the surface of colonic epithelial cells. In healthy controls, the staining of occludin was intense and continuous, while faint and discontinuous staining was observed in the colon tissue of IBS-D patients (Fig. 2A). Western blot analysis showed a protein band at $\sim 65 \mathrm{kDa}$ for occludin. Relative occludin expression in the rectosigmoid colon was lower in patients with IBS-D compared with that in healthy controls $(0.38 \pm 0.14$ vs. $1.24 \pm 0.39, \mathrm{P}<0.001)$ (Fig. 2B).

Correlation between cytokine production and occludin expression in IBS-D patients. In patients with IBS-D, occludin 
Table II. Association of cytokine production in peripheral blood mononuclear cells of patients with diarrheic irritable bowel syndrome and anxiety as well as depression scores.

\begin{tabular}{lccc}
\hline Score & TNF- $\alpha(\mathrm{pg} / \mathrm{ml})$ & IL-8 $(\mathrm{pg} / \mathrm{ml})$ & IL-10 $(\mathrm{pg} / \mathrm{ml})$ \\
\hline SAS & & & \\
$\geq 50(\mathrm{n}=17)$ & $86.1 \pm 23.4$ & $169.3 \pm 30.7$ & $82.6 \pm 25.7$ \\
$<50(\mathrm{n}=25)$ & $80.0 \pm 21.1$ & $142.0 \pm 36.7^{\mathrm{b}}$ & $89.2 \pm 24.3$ \\
SDS & & & $83.5 \pm 25.3$ \\
$\geq 50(\mathrm{n}=20)$ & $93.3 \pm 22.0$ & $168.5 \pm 35.2$ & $91.2 \pm 18.7$ \\
$<50(\mathrm{n}=22)$ & $73.6 \pm 18.7^{\mathrm{a}}$ & $139.1 \pm 32.4^{\mathrm{a}}$ &
\end{tabular}

Values are presented as the mean \pm standard deviation. ${ }^{\mathrm{P}}<0.05$ compared with $\mathrm{SDS}$ score $\geq 50$; ${ }^{\mathrm{b}} \mathrm{P}<0.05$ compared with $\mathrm{SAS} \geq 50$. SAS, self-rating anxiety scale; SDS, self-rating depression scale; IL, interleukin; TNF, tumor necrosis factor.
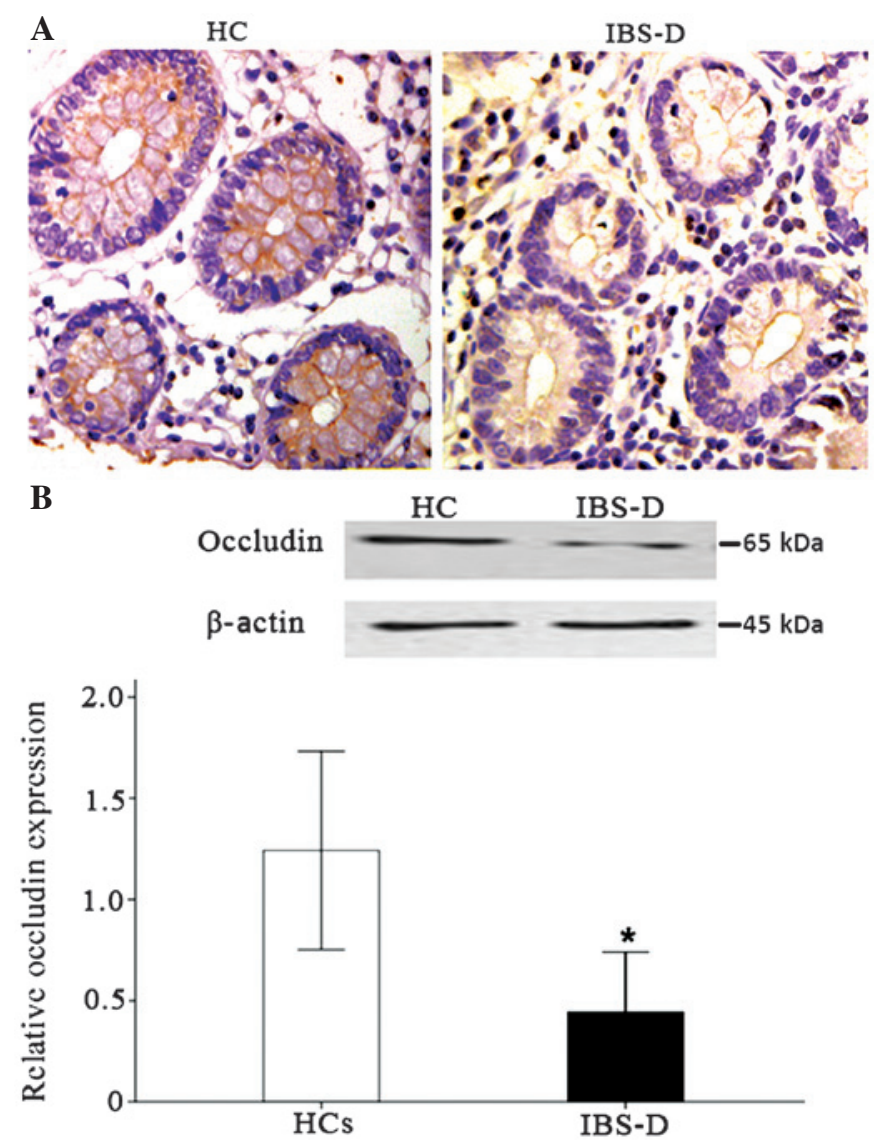

Figure 2. Expression of tight junction protein occludin in the rectosigmoid colonal tissues of HCs and IBS-D patients. (A) Immunostaining of the tight junction protein occludin in the rectosigmoid colonal tissues of HCs and IBS-D patients (magnification, x200). Brown and yellow staining indicate positivity for occludin. (B) Western blot analysis of tight junction protein occludin in the rectosigmoid colonal tissues of HCs and IBS-D patients. Analysis showed a protein band at $\sim 65 \mathrm{kDa}$ for the tight junction protein occludin. The same experiment was repeated three times. Relative occludin expression was quantified by integral optical density is expressed as the mean \pm standard deviation in HCs and IBS-D patients. ${ }^{*} \mathrm{P}<0.01$. IBS-D, irritable bowel syndrome with diarrhea; $\mathrm{HC}$, healthy control.

expression was negatively correlated with TNF- $\alpha$ and IL-8 production $(\mathrm{r}=-0.34, \mathrm{P}=0.028 ; \mathrm{r}=-0.52, \mathrm{P}<0.001$, respectively) (Fig. 3A and B). However, there was no correlation between occludin and IL-10 production ( $\mathrm{r}=0.05, \mathrm{P}=0.748)$ (Fig. $3 \mathrm{C})$.
Correlation between cytokine production and abdominal symptoms score in patients with IBS-D. The mean abdominal symptoms score was $11.17 \pm 5.06$ for IBS-D patients and was positively correlated with TNF- $\alpha$ and IL- 8 production $(r=0.74$, $\mathrm{P}<0.001 ; \mathrm{r}=0.55, \mathrm{P}<0.001$, respectively); however, it was negatively correlated with IL-10 production $(\mathrm{r}=-0.80, \mathrm{P}<0.001)$ (Fig. 4A-C).

Psychological distress and correlation with cytokine levels in IBS-D patients. Among the 42 patients, 20 (66.7\%) with an SDS score $\geq 50$ were diagnosed with depression and 17 (40.4\%) had an SAS score $\geq 50$ and were considered to have anxiety. IBS-D patients with an SDS score $\geq 50$ had significantly higher TNF- $\alpha(\mathrm{P}=0.004)$ and IL-8 levels $(\mathrm{P}=0.008)$ than those with an SDS score $<50$. IBS-D patients with an SAS score $\geq 50$ showed significantly higher IL-8 expression compared with those with an SAS score $<50(\mathrm{P}=0.016)$. However, there was no statistical difference in IL-10 expression between patients with SAS or SDS score $\geq 50$ and those with lower scores $(\mathrm{P}=0.385$; $\mathrm{P}=0.275$ ) (Table II).

\section{Discussion}

IBS-D, a subgroup of IBS, is common in males and females (17). Evidence showed that immune activation was an important event in the development of IBS (18). PBMCs, including lymphocytes, leukocytes and macrophages, have key roles in immune activation and have been used to investigate the specific cytokines that can indicate the disease stages (19). In the present study, levels of the pro-inflammatory cytokines TNF- $\alpha$ and IL- 8 and those of the anti-inflammatory cytokine IL-10 were assessed in PBMCs from IBS-D patients. The results showed that TNF- $\alpha$ and IL- 8 production were significantly greater in PBMCs from IBS-D patients than those in healthy controls, while IL-10 production was significantly decreased in IBS-D patients. These findings were consistent with the widely accepted mechanism of IBS, namely that the imbalance of cytokines involved in inflammatory response results in persisting inflammation (5). A previous study showed that the frequency of the high-producer genotype for IL-10 was significantly lower in patients with IBS compared with that in healthy ones, which may contribute to the progression of inflammation in IBS (5). 

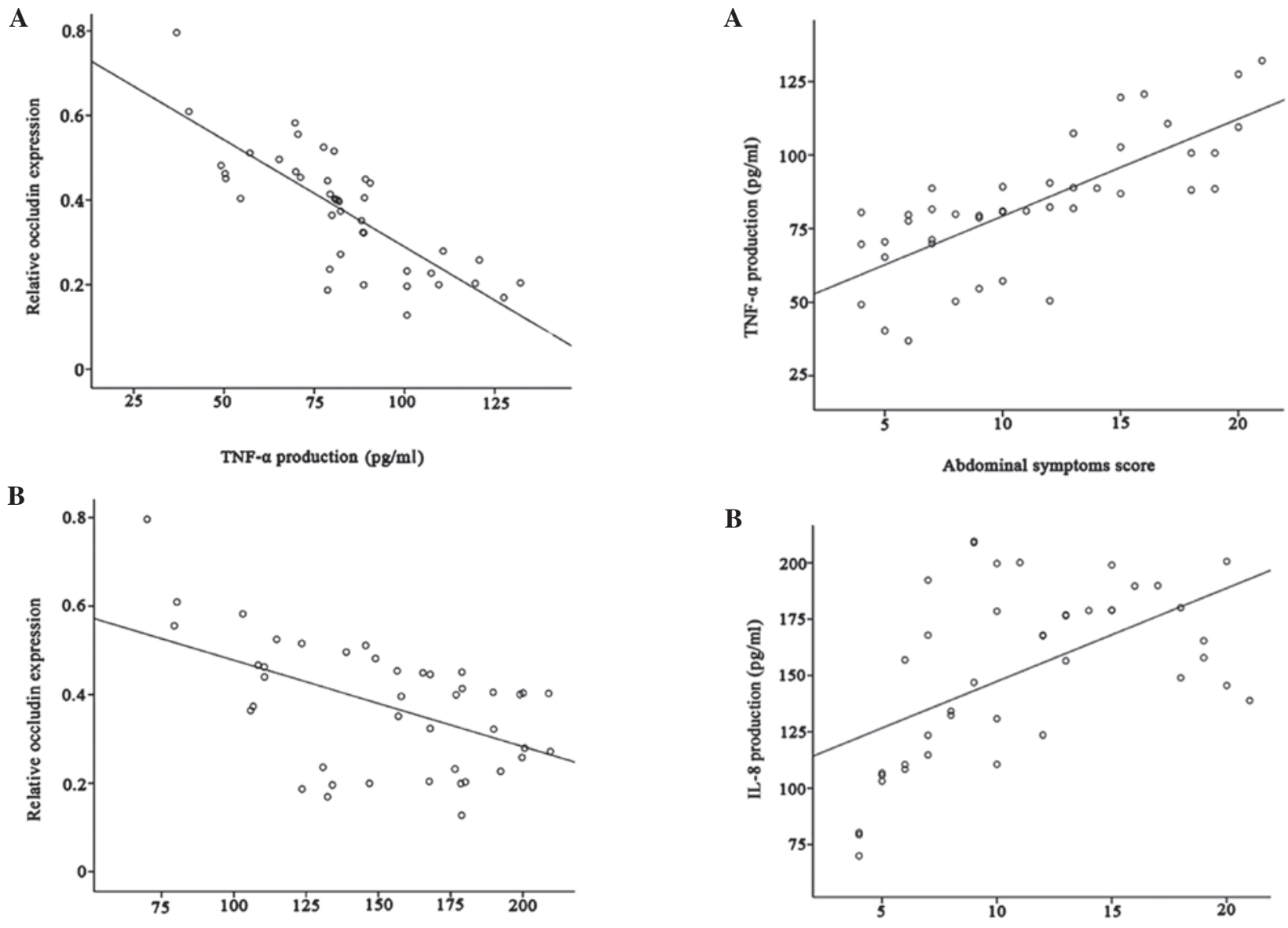

II-8 production $(\mathrm{pg} / \mathrm{ml})$
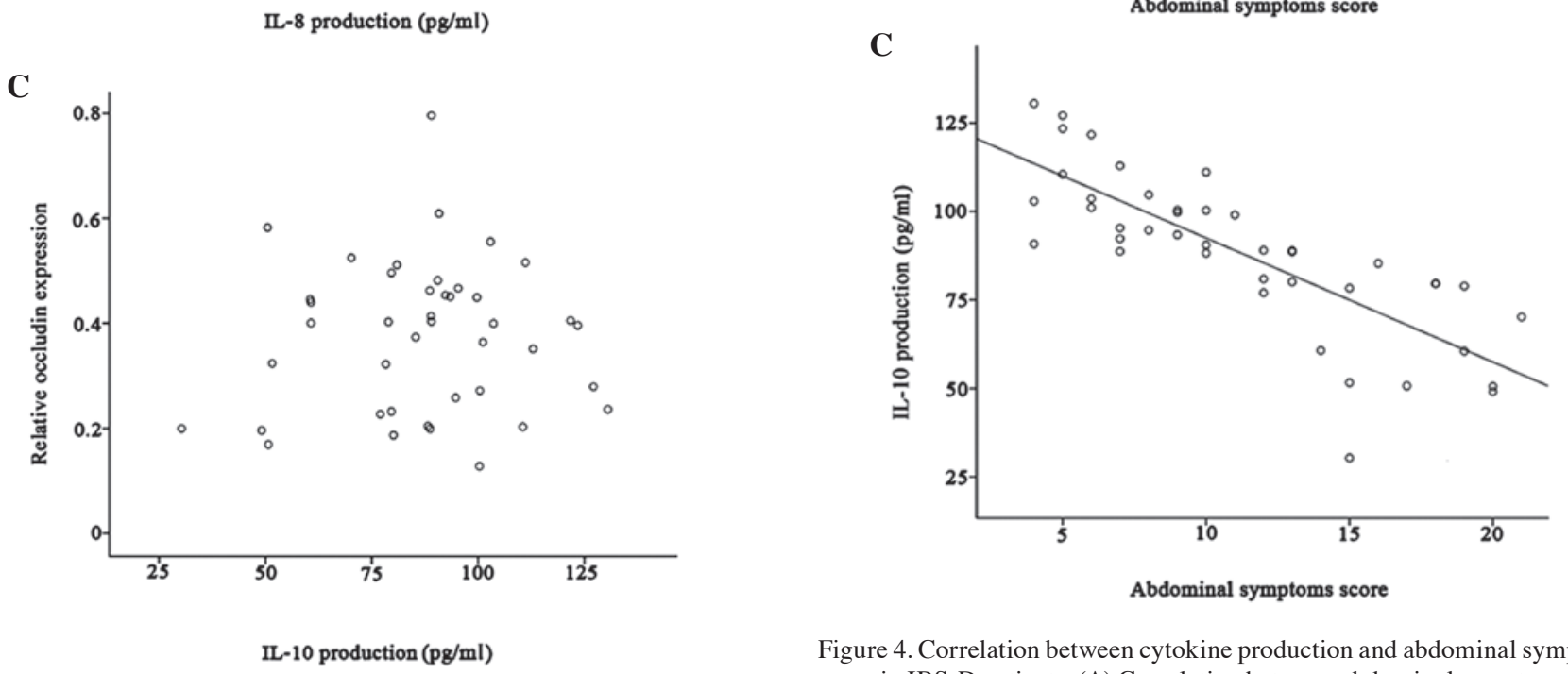

Figure 3. Correlation of cytokine production and occludin expression in IBS-D patients. (A) Correlation between production of TNF- $\alpha$ and occludin in IBS-D patients. Occludin expression was negatively correlated with the production of TNF- $\alpha$ in PBMCs ( $\mathrm{r}=-0.34, \mathrm{P}=0.028)$. (B) Correlation between IL-8 in and occludin expression in IBS-D patients. Occludin expression was negatively correlated to the production of IL-8 in PBMCs $(r=-0.52$, $\mathrm{P}<0.001)$. (C) Correlation between the expression of IL-10 and occludin in IBS-D patients. Occludin expression was not correlated with IL-10 production $(r=0.05, P=0.748)$. TNF- $\alpha$, tumor necrosis factor alpha; IL, interleukin; IBS-D, irritable bowel syndrome with diarrhea; PBMC, peripheral blood mononuclear cell.

Although a previous study reported that the increasing activity of faecal serine protease from patients with HBS-D

Figure 4. Correlation between cytokine production and abdominal symptoms score in IBS-D patients. (A) Correlation between abdominal symptoms score and TNF- $\alpha$ production in IBS-D patients $(\mathrm{r}=0.74, \mathrm{P}<0.001)$. (B) Correlation between abdominal symptoms score and IL-8 production in IBS-D patients $(\mathrm{r}=0.55, \mathrm{P}<0.001)$. (C) Correlation between abdominal symptoms score and IL-10 production in IBS-D patients $(r=-0.80, \mathrm{P}<0.001)$. TNF- $\alpha$, tumor necrosis factor alpha; IL, interleukin; IBS-D, irritable bowel syndrome with diarrhea.

was able to induce a marked internalization of tight junction proteins (20), the present study showed that occludin was restricted to the surface of colonic epithelial cells in HCs and IBS-D patients without intensive internalization. The present study also observed the dysfunction of the tight junction protein occludin in IBS-D patients by immunohistochemistry 
and western blot analysis. The immunohistochemical staining of occludin was faint and discontinuous in IBS-D patients and occludin protein was significantly decreased compared with that in healthy controls. Recent evidence also suggested that occudin expression was decreased in IBS-D patients and the levels of occudin were negatively correlated with the intensity of symptoms (21). Occludin is one of the tight junction proteins, and an important component of intestinal mucosa. Patients with occludin disruption also present with impaired intestinal permeability and increased stool frequency (22). In the present study, reduced occludin levels indicated that the tight junction was disrupted in IBS-D patients. Therefore, although the occludin distribution appeared normal under colonoscopy and conventional histology, the intestinal mucosal barrier of the IBS-D patients had become disrupted.

It is well documented that IBS patients show an increased number of immunocompetent cells in the mucosal immune response (23). Thus, it was hypothesized that the increased mucosal immunocytes, along with elevated levels of systemic cytokines, including TNF- $\alpha$ and IL-8, contributed to mucosal barrier disruption. The findings of the present study indicated that occludin expression was negatively correlated with TNF- $\alpha$ and IL- 8 production, but was not correlated with IL-10 production. In addition, the present study indicated that the mean symptom score of IBS-D patients was positively correlated with TNF- $\alpha$ and IL-8 production, but was negatively correlated with IL-10 production. The peripheral inflammation is characterized by elevated pain sensitivity, which is mediated by the release of inflammatory cytokines and growth factors (24). TNF- $\alpha$ was reported to contribute to inflammatory sensory hypersensitivity (24). Therefore, elevated levels of cytokines may be sufficient to sensitize sensory nerve terminals, which may have resulted in a decreased threshold for visceral sensitivity in IBS patients and explain the high abdominal symptoms score in patients with IBS-D.

In addition, the present study also found significant associations between psychological distress and cytokine production. IBS patients with psychological distress trended to show higher production of pro-inflammatory cytokines (TNF- $\alpha$ and IL-8) and lower levels of the anti-inflammatory cytokine IL-10. Similar results were reported in a recent study that indicated that the levels of pro-inflammatory cytokine IL-1 $\beta$ were significantly higher in IBS-D patients with anxiety and depression and that the IL-10 levels were significantly lower compared with those in IBS-D patients without anxiety and depression ones (25). These results suggested that psychological distress may cause changes in pro-inflammatory and anti-inflammatory cytokine expression in IBS-D patients, which may contribute to the occurrence or aggravation of IBS.

Psychiatric and psychological factors are closely associated with the mechanism of IBS. The effect of psychological factors on the symptoms and outcome of IBS is becoming increasingly recognized. Studies have indicated that psychiatric and psychological factors are independent risks for developing IBS (26). Psychological stress can contribute to the initiation and reactivation of experimental gastrointestinal inflammation and increase the intestinal permeability $(27,28)$. Piche et al (10) also found that the number of IBS mast cells was significantly correlated with the Fatigue Impact Scale score and the Beck Depression Inventory score. The results of the present study demonstrated that psychological factors may alter immunological function and lead to the increased secretion of pro-inflammatory cytokines.

However, it must be pointed out that the present study has certain limitations: Due to the small cohort size used in the present study, further studies using a large number of samples are required to confirm the results. Furthermore, different subgroups of IBS and additional cytokines should be studied to better elucidate the pathological mechanisms of IBS.

In conclusion, the present study demonstrated that the balance of cytokine production was impaired in IBS-D patients. The imbalanced cytokines expression evoked colonic epithelial barrier dysfunction and may be associated with abdominal symptoms and psychological disorders.

\section{Acknowledgements}

This study was funded by a grant from the National Science Foundation of China (NSFC; no. 30971336). The authors appreciate the considerable assistance from the Gastroenterology Kinetic Laboratory and the Key Laboratory of Cardiovascular Remodeling and Function Research at Qilu Hospital, Shandong University.

\section{References}

1. Schmulson MW and Chang L: Diagnostic approach to the patient with irritable bowel syndrome. Am J Med 107: 20S-26S, 1999.

2. Holten KB, Wetherington A and Bankston L: Diagnosing the patient with abdominal pain and altered bowel habits: is it irritable bowel syndrome? Am Fam Physician 67: 2157-2164, 2003.

3. Andresen V and Camilleri M: Irritable bowel syndrome: recent and novel therapeutic approaches. Drugs 66: 1073-1088, 2006.

4. van der Veek PP, van den Berg M, de Kroon YE, Verspaget HW and Masclee AA: Role of tumor necrosis factor-alpha and interleukin-10 gene polymorphisms in irritable bowel syndrome. Am J Gastroenterol 100: 2510-2516, 2005.

5. Gonsalkorale WM, Perrey C, Pravica V, Whorwell PJ and Hutchinson IV: Interleukin 10 genotypes in irritable bowel syndrome: evidence for an inflammatory component? Gut 52: 91-93, 2003.

6. Nielsen $\mathrm{OH}$, Rüdiger N, Gaustadnes M and Horn T: Intestinal interleukin-8 concentration and gene expression in inflammatory bowel disease. Scand J Gastroenterol 32: 1028-1034, 1997.

7. Coëffier M, Gloro R, Boukhettala N, et al: Increased proteasome-mediated degradation of occludin in irritable bowel syndrome. Am J Gastroenterol 105: 1181-1188, 2010.

8. Simrén M, Abrahamsson H, Svedlund J and Björnsson E: Quality of life in patients with irritable bowel syndrome seen in referral centers versus primary care: the impact of gender and predominant bowel pattern. Scand J Gastroenterol 36: 545-552, 2001.

9. Piche T, Huet PM, Gelsi E, et al: Fatigue in irritable bowel syndrome: characterization and putative role of leptin. Eur J Gastroenterol Hepatol 19: 237-243, 2007.

10. Piche T, Saint-Paul MC, Dainese R, et al: Mast cells and cellularity of the colonic mucosa correlated with fatigue and depression in irritable bowel syndrome. Gut 57: 468-473, 2008.

11. Drossman DA and Dumitrascu DL: Rome III: New standard for functional gastrointestinal disorders. J Gastrointestin Liver Dis 15: 237-241, 2006.

12. Lewis SJ and Heaton KW: Stool form scale as a useful guide to intestinal transit time. Scand J Gastroenterol 32: 920-924, 1997.

13. Endo Y, Yoshizawa M, Fukudo S, Sasaki M and Hongo M: Panic disorder in irritable bowel syndrome. Jpn J Psychosom Med 40: 339-346, 2000 .

14. Liebregts T, Adam B, Bredack C, et al: Immune activation in patients with irritable bowel syndrome. Gastroenterology 132: 913-920, 2007.

15. Zung WW: A rating instrument for anxiety disorders. Psychosomatics 12: 371-379, 1971. 
16. Zung WW: A self-rating depression scale. Arch Gen Psychiatry 12: 63-70, 1965.

17. Chang L, Ameen VZ, Dukes GE, McSorley DJ, Carter EG and Mayer EA: A dose-ranging, phase II study of the efficacy and safety of alosetron in men with diarrhea-predominant IBS. Am J Gastroenterol 100: 115-123, 2005.

18. Ohman L and Simrén M: Pathogenesis of IBS: role of inflammation, immunity and neuroimmune interactions. Nat Rev Gastroenterol Hepatol 7: 163-173, 2010.

19. Nakamura M, Saito H, Kasanuki J, Tamura Y and Yoshida S Cytokine production in patients with inflammatory bowel disease. Gut 33: 933-937, 1992.

20. Gecse K, Roka R, Ferrier L, et al: Increased faecal serine protease activity in diarrhoeic IBS patients: a colonic lumenal factor impairing colonic permeability and sensitivity. Gut 57: 591-599, 2008.

21. Bertiaux-Vandaele N, Youmba SB, Belmonte L, et al: The expression and the cellular distribution of the tight junction proteins are altered in irritable bowel syndrome patients with differences according to the disease subtype. Am J Gastroenterol 106: 2165-2173, 2011.

22. Dunlop SP, Hebden J, Campbell E, et al: Abnormal intestinal permeability in subgroups of diarrhea-predominant irritable bowel syndromes. Am J Gastroenterol 101: 1288-1294, 2006.
23. Chadwick VS, Chen W, Shu D, et al: Activation of the mucosal immune system in irritable bowel syndrome. Gastroenterology 122: 1778-1783, 2002.

24. Woolf C, Allchorne A, Safieh-Garabedian B and Poole S: Cytokines, nerve growth factor and inflammatory hyperalgesia: the contribution of tumour necrosis factor alpha. Br J Pharmacol 121: 417-424, 1997.

25. Gao J: Correlation between anxiety-depression status and cytokines in diarrhea-predominant irritable bowel syndrome. Exp Ther Med 6: 93-96, 2013.

26. Nicholl BI, Halder SL, Macfarlane GJ, et al: Psychosocial risk markers for new onset irritable bowel syndrome - results of a large prospective population-based study. Pain 137: 147-155, 2008.

27. Qiu BS, Vallance BA, Blennerhassett PA and Collins SM: The role of CD4+ lymphocytes in the susceptibility of mice to stress-induced reactivation of experimental colitis. Nat Med 5: 1178-1182, 1999.

28. Kiliaan AJ, Saunders PR, Bijlsma PB, et al: Stress stimulates transepithelial macromolecular uptake in rat jejunum. Am J Physiol 275 (5 Pt 1): G1037-G1044, 1998. 